\title{
Triangle-free distance-regular graphs
}

\author{
Yeh-jong Pan • Min-hsin Lu • Chih-wen Weng
}

Received: 16 March 2006 / Accepted: 2 April 2007 /

Published online: 24 May 2007

(C) Springer Science+Business Media, LLC 2007

\begin{abstract}
Let $\Gamma$ denote a distance-regular graph with diameter $d \geq 3$. By a parallelogram of length 3, we mean a 4-tuple $x y z w$ consisting of vertices of $\Gamma$ such that $\partial(x, y)=\partial(z, w)=1, \partial(x, z)=3$, and $\partial(x, w)=\partial(y, w)=\partial(y, z)=2$, where $\partial$ denotes the path-length distance function. Assume that $\Gamma$ has intersection numbers $a_{1}=0$ and $a_{2} \neq 0$. We prove that the following (i) and (ii) are equivalent. (i) $\Gamma$ is $Q$-polynomial and contains no parallelograms of length 3; (ii) $\Gamma$ has classical parameters $(d, b, \alpha, \beta)$ with $b<-1$. Furthermore, suppose that (i) and (ii) hold. We show that each of $b(b+1)^{2}(b+2) / c_{2},(b-2)(b-1) b(b+1) /\left(2+2 b-c_{2}\right)$ is an integer and that $c_{2} \leq b(b+1)$. This upper bound for $c_{2}$ is optimal, since the Hermitian forms graph $\operatorname{Her}_{2}(d)$ is a triangle-free distance-regular graph that satisfies $c_{2}=b(b+1)$.
\end{abstract}

Keywords Distance-regular graph · $Q$-polynomial · Classical parameters

\section{Introduction}

Let $\Gamma$ denote a distance-regular graph with diameter $d \geq 3$ (see Sect. 2 for formal definitions). It is known that if $\Gamma$ has classical parameters, then $\Gamma$ is $Q$-polynomial [2, Corollary 8.4.2]. The converse is not true, since an ordinary $n$-gon has the $Q$ polynomial property, but is without classical parameters [2, Table 6.6]. Many authors prove the converse under various additional assumptions. Indeed, assume that $\Gamma$ is $Q$-polynomial. Then Brouwer, Cohen, and Neumaier [2, Theorem 8.5.1] show that

Work partially supported by the National Science Council of Taiwan, R.O.C.

Y.-j. Pan ( $\varangle) \cdot$ M.-h. Lu · C.-w. Weng

Department of Applied Mathematics, National Chiao Tung University,

1001 Ta Hsueh Road Hsinchu, Taiwan 30010, Taiwan

e-mail: yjp.9222803@nctu.edu.tw

C.-w. Weng

e-mail: weng@math.nctu.edu.tw 
if $\Gamma$ is a near polygon with the intersection number $a_{1} \neq 0$, then $\Gamma$ has classical parameters. Weng generalizes this result with a weaker assumption, without kites of length 2 or length 3 in $\Gamma$, to replace the near polygon assumption [10, Lemma 2.4]. For the complement case $a_{1}=0$, Weng shows that $\Gamma$ has classical parameters if (i) $\Gamma$ contains no parallelograms of length 3 and no parallelograms of length 4; (ii) $\Gamma$ has the intersection number $a_{2} \neq 0$; and (iii) $\Gamma$ has diameter $d \geq 4$ [11, Theorem 2.11]. Our first theorem improves the above result.

Theorem 1.1 Let $\Gamma$ denote a distance-regular graph with diameter $d \geq 3$ and intersection numbers $a_{1}=0$ and $a_{2} \neq 0$. Then the following (i)-(iii) are equivalent:

(i) $\Gamma$ is Q-polynomial and contains no parallelograms of length 3.

(ii) $\Gamma$ is $Q$-polynomial and contains no parallelograms of any length $i$ for $3 \leq i \leq d$.

(iii) $\Gamma$ has classical parameters $(d, b, \alpha, \beta)$ with $b<-1$.

Many authors study distance-regular graph $\Gamma$ with $a_{1}=0$ and other additional assumptions. For example, Miklavič assumes that $\Gamma$ is $Q$-polynomial and shows that $\Gamma$ is 1-homogeneous [6]; Koolen and Moulton assume that $\Gamma$ has degree 8, 9, or 10 and show that there are finitely many such graphs [5]; Jurišić, Koolen, and Miklavič assume that $\Gamma$ has an eigenvalue with multiplicity equal to the valency, $a_{2} \neq 0$, and the diameter $d \geq 4$ to show that $a_{4}=0$ and $\Gamma$ is 1-homogeneous [4]. In the second theorem, we assume that $\Gamma$ has classical parameters and obtain the following:

Theorem 1.2 With the notation and assumptions of Theorem 1.1, suppose that (i)-(iii) hold. Then each of

$$
\frac{b(b+1)^{2}(b+2)}{c_{2}}, \quad \frac{(b-2)(b-1) b(b+1)}{2+2 b-c_{2}}
$$

is an integer. Moreover,

$$
c_{2} \leq b(b+1)
$$

To conclude the paper, we give a class of triangle-free distance-regular graphs, each satisfying the equality in (1.2).

\section{Preliminaries}

In this section, we review some definitions and basic concepts concerning distanceregular graphs. See Bannai and Ito [1] or Terwilliger [8] for more background information.

Let $\Gamma=(X, R)$ denote a finite undirected connected graph without loops or multiple edges with vertex set $X$, edge set $R$, distance function $\partial$, and diameter $d:=$ $\max \{\partial(x, y) \mid x, y \in X\}$.

For a vertex $x \in X$ and $0 \leq i \leq d$, set $\Gamma_{i}(x)=\{y \in X \mid \partial(x, y)=i\} . \Gamma$ is said to be distance-regular whenever for all integers $0 \leq h, i, j \leq d$ and all vertices $x, y \in X$ with $\partial(x, y)=h$, the number

$$
p_{i j}^{h}=\left|\left\{z \in X \mid z \in \Gamma_{i}(x) \cap \Gamma_{j}(y)\right\}\right|
$$


is independent of $x, y$. The constants $p_{i j}^{h}$ are known as the intersection numbers of $\Gamma$. For convenience, set $c_{i}:=p_{1 i-1}^{i}$ for $1 \leq i \leq d, a_{i}:=p_{1 i}^{i}$ for $0 \leq i \leq d, b_{i}:=p_{1 i+1}^{i}$ for $0 \leq i \leq d-1$, and put $b_{d}:=0, c_{0}:=0, k:=b_{0}$. Note that $k$ is called the valency of $\Gamma$. From the definition of $p_{i j}^{h}$ it is immediate that $b_{i} \neq 0$ for $0 \leq i \leq d-1$ and $c_{i} \neq 0$ for $1 \leq i \leq d$. Moreover,

$$
k=a_{i}+b_{i}+c_{i} \quad \text { for } 0 \leq i \leq d .
$$

From now on we assume that $\Gamma$ is distance-regular with diameter $d \geq 3$.

Let $\mathbb{R}$ denote the real number field. Let $\operatorname{Mat}_{X}(\mathbb{R})$ denote the algebra of all matrices over $\mathbb{R}$ with the rows and columns indexed by the elements of $X$. For $0 \leq i \leq d$, let $A_{i}$ denote the matrix in $\operatorname{Mat}_{X}(\mathbb{R})$ defined by the rule

$$
\left(A_{i}\right)_{x y}=\left\{\begin{array}{ll}
1 & \text { if } \partial(x, y)=i, \\
0 & \text { if } \partial(x, y) \neq i
\end{array} \quad \text { for } x, y \in X .\right.
$$

We call $A_{i}$ the distance matrices of $\Gamma$. We have

$$
\begin{aligned}
& A_{0}=I, \\
& A_{0}+A_{1}+\cdots+A_{d}=J, \quad \text { where } J=\text { all 1's matrix, } \\
& A_{i}^{\mathrm{t}}=A_{i} \quad \text { for } 0 \leq i \leq d, \text { where } A_{i}^{\mathrm{t}} \text { means the transpose of } A_{i}, \\
& A_{i} A_{j}=\sum_{h=0}^{d} p_{i j}^{h} A_{h} \quad \text { for } 0 \leq i, j \leq d, \\
& A_{i} A_{j}=A_{j} A_{i} \quad \text { for } 0 \leq i, j \leq d .
\end{aligned}
$$

Let $M$ denote the subspace of $\operatorname{Mat}_{X}(\mathbb{R})$ spanned by $A_{0}, A_{1}, \ldots, A_{d}$. Then $M$ is a commutative subalgebra of $\operatorname{Mat}_{X}(\mathbb{R})$ and is known as the Bose-Mesner algebra of $\Gamma$. By [2, p. 59, 64], $M$ has a second basis $E_{0}, E_{1}, \ldots, E_{d}$ such that

$$
\begin{aligned}
& E_{0}=|X|^{-1} J, \\
& E_{i} E_{j}=\delta_{i j} E_{i} \quad \text { for } 0 \leq i, j \leq d, \\
& E_{0}+E_{1}+\cdots+E_{d}=I, \\
& E_{i}^{\mathrm{t}}=E_{i} \quad \text { for } 0 \leq i \leq d .
\end{aligned}
$$

The $E_{0}, E_{1}, \ldots, E_{d}$ are known as the primitive idempotents of $\Gamma$, and $E_{0}$ is known as the trivial idempotent. Let $E$ denote any primitive idempotent of $\Gamma$. Then we have

$$
E=|X|^{-1} \sum_{i=0}^{d} \theta_{i}^{*} A_{i}
$$

for some $\theta_{0}^{*}, \theta_{1}^{*}, \ldots, \theta_{d}^{*} \in \mathbb{R}$ called the dual eigenvalues associated with $E$.

Set $V=\mathbb{R}^{|X|}$ (column vectors) and view the coordinates of $V$ as being indexed by $X$. Then the Bose-Mesner algebra $M$ acts on $V$ by left multiplication. We call $V$ 
the standard module of $\Gamma$. For each vertex $x \in X$, set

$$
\hat{x}=(0,0, \ldots, 0,1,0, \ldots, 0)^{\mathrm{t}}
$$

where the 1 is in coordinate $x$. Also, let $\langle$,$\rangle denote the dot product$

$$
\langle u, v\rangle=u^{\mathrm{t}} v \quad \text { for } u, v \in V
$$

Then referring to the primitive idempotent $E$ in (2.11), from (2.10-2.13) we compute that, for $x, y \in X$,

$$
\langle E \hat{x}, \hat{y}\rangle=|X|^{-1} \theta_{i}^{*}
$$

where $i=\partial(x, y)$.

Let $\circ$ denote the entry-wise multiplication in $\operatorname{Mat}_{X}(\mathbb{R})$. Then

$$
A_{i} \circ A_{j}=\delta_{i j} A_{i} \quad \text { for } 0 \leq i, j \leq d,
$$

so $M$ is closed under $\circ$. Thus there exists $q_{i j}^{k} \in \mathbb{R}$ for $0 \leq i, j, k \leq d$ such that

$$
E_{i} \circ E_{j}=|X|^{-1} \sum_{k=0}^{d} q_{i j}^{k} E_{k} \quad \text { for } 0 \leq i, j \leq d .
$$

$\Gamma$ is said to be $Q$-polynomial with respect to the given ordering $E_{0}, E_{1}, \ldots, E_{d}$ of the primitive idempotents if, for all integers $0 \leq h, i, j \leq d, q_{i j}^{h}=0$ (resp. $q_{i j}^{h} \neq 0$ ) whenever one of $h, i, j$ is greater than (resp. equal to) the sum of the other two. Let $E$ denote any primitive idempotent of $\Gamma$. Then $\Gamma$ is said to be $Q$-polynomial with respect to $E$ whenever there exists an ordering $E_{0}, E_{1}=E, \ldots, E_{d}$ of the primitive idempotents of $\Gamma$ with respect to which $\Gamma$ is $Q$-polynomial. If $\Gamma$ is $Q$-polynomial with respect to $E$, then the associated dual eigenvalues are distinct [7, p. 384].

The following theorem about the $Q$-polynomial property will be used in this paper.

Theorem 2.1 [8, Theorem 3.3] Assume that $\Gamma$ is $Q$-polynomial with respect to a primitive idempotent $E$, and let $\theta_{0}^{*}, \ldots, \theta_{d}^{*}$ denote the corresponding dual eigenvalues. Then the following (i)-(ii) hold:

(i) For all integers $1 \leq h \leq d, 0 \leq i, j \leq d$ and for all $x, y \in X$ such that $\partial(x, y)=h$,

$$
\sum_{\substack{z \in X, \partial(x, z)=i \\ \partial(y, z)=j}} E \hat{z}-\sum_{\substack{z \in X, \partial(x, z)=j \\ \partial(y, z)=i}} E \hat{z}=p_{i j}^{h} \frac{\theta_{i}^{*}-\theta_{j}^{*}}{\theta_{0}^{*}-\theta_{h}^{*}}(E \hat{x}-E \hat{y}) .
$$

(ii) For an integer $3 \leq i \leq d$,

$$
\theta_{i-2}^{*}-\theta_{i-1}^{*}=\sigma\left(\theta_{i-3}^{*}-\theta_{i}^{*}\right)
$$

for an appropriate $\sigma \in \mathbb{R} \backslash\{0\}$. 
$\Gamma$ is said to have classical parameters $(d, b, \alpha, \beta)$ whenever the intersection numbers of $\Gamma$ satisfy

$$
\begin{aligned}
& c_{i}=\left[\begin{array}{l}
i \\
1
\end{array}\right]\left(1+\alpha\left[\begin{array}{c}
i-1 \\
1
\end{array}\right]\right) \quad \text { for } 0 \leq i \leq d, \\
& b_{i}=\left(\left[\begin{array}{l}
d \\
1
\end{array}\right]-\left[\begin{array}{l}
i \\
1
\end{array}\right]\right)\left(\beta-\alpha\left[\begin{array}{l}
i \\
1
\end{array}\right]\right) \quad \text { for } 0 \leq i \leq d,
\end{aligned}
$$

where

$$
\left[\begin{array}{l}
i \\
1
\end{array}\right]:=1+b+b^{2}+\cdots+b^{i-1} .
$$

Suppose that $\Gamma$ has classical parameters $(d, b, \alpha, \beta)$. Combining (2.17-2.19) with (2.1), we have

$$
\begin{aligned}
a_{i} & =\left[\begin{array}{l}
i \\
1
\end{array}\right]\left(\beta-1+\alpha\left(\left[\begin{array}{l}
d \\
1
\end{array}\right]-\left[\begin{array}{l}
i \\
1
\end{array}\right]-\left[\begin{array}{c}
i-1 \\
1
\end{array}\right]\right)\right) \\
& =\left[\begin{array}{l}
i \\
1
\end{array}\right]\left(a_{1}+\alpha\left(1-\left[\begin{array}{l}
i \\
1
\end{array}\right]-\left[\begin{array}{c}
i-1 \\
1
\end{array}\right]\right)\right) \text { for } 0 \leq i \leq d .
\end{aligned}
$$

Note that if $\Gamma$ has classical parameters $(d, b, \alpha, \beta)$ and $d \geq 3$, then $b$ is an integer and $b \neq 0,-1$ [2, Proposition 6.2.1]. $\Gamma$ is said to have classical parameters if $\Gamma$ has classical parameters $(d, b, \alpha, \beta)$ for some constants $d, b, \alpha, \beta$. It is shown that a distance-regular graph with classical parameters has the $Q$-polynomial property [2, Theorem 8.4.1]. Terwilliger generalizes this to the following:

Theorem 2.2 [8, Theorem 4.2] Let $\Gamma$ denote a distance-regular graph with diameter $d \geq 3$. Choose $b \in \mathbb{R} \backslash\{0,-1\}$. Then the following (i)-(ii) are equivalent:

(i) $\Gamma$ is $Q$-polynomial with associated dual eigenvalues $\theta_{0}^{*}, \theta_{1}^{*}, \ldots, \theta_{d}^{*}$ satisfying

$$
\theta_{i}^{*}-\theta_{0}^{*}=\left(\theta_{1}^{*}-\theta_{0}^{*}\right)\left[\begin{array}{l}
i \\
1
\end{array}\right] b^{1-i} \quad \text { for } 1 \leq i \leq d .
$$

(ii) $\Gamma$ has classical parameters $(d, b, \alpha, \beta)$ for some real constants $\alpha, \beta$.

Pick an integer $2 \leq i \leq d$. By a parallelogram of length $i$ in $\Gamma$ we mean a 4-tuple $x y z w$ of vertices of $X$ such that (see Fig. 1)

Fig. 1 A parallelogram of length $i$

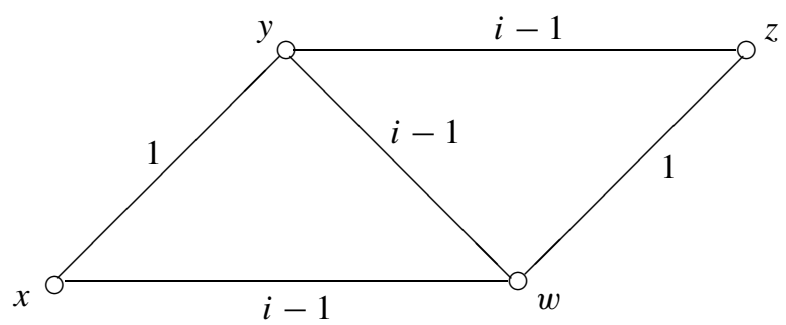




$$
\begin{aligned}
& \partial(x, y)=\partial(z, w)=1, \quad \partial(x, z)=i, \\
& \partial(x, w)=\partial(y, w)=\partial(y, z)=i-1 .
\end{aligned}
$$

\section{Proof of Theorem 1.1}

In this section we prove our first main theorem. We start with a lemma.

Lemma 3.1 [6, Theorem 5.2(i)] Let $\Gamma$ denote a $Q$-polynomial distance-regular graph with diameter $d \geq 3$ and intersection number $a_{1}=0$. Fix an integer $i$ for $2 \leq i \leq d$ and three vertices $x, y, z$ such that

$$
\partial(x, y)=1, \quad \partial(y, z)=i-1, \quad \partial(x, z)=i .
$$

Then the quantity

$$
s_{i}(x, y, z):=\left|\Gamma_{i-1}(x) \cap \Gamma_{i-1}(y) \cap \Gamma_{1}(z)\right|
$$

is equal to

$$
a_{i-1} \frac{\left(\theta_{0}^{*}-\theta_{i-1}^{*}\right)\left(\theta_{2}^{*}-\theta_{i}^{*}\right)-\left(\theta_{1}^{*}-\theta_{i-1}^{*}\right)\left(\theta_{1}^{*}-\theta_{i}^{*}\right)}{\left(\theta_{0}^{*}-\theta_{i-1}^{*}\right)\left(\theta_{i-1}^{*}-\theta_{i}^{*}\right)} .
$$

In particular, (3.1) is independent of the choice of the vertices $x, y, z$.

Proof Let $s_{i}(x, y, z)$ denote the expression in (3.1) and set

$$
\ell_{i}(x, y, z)=\left|\Gamma_{i}(x) \cap \Gamma_{i-1}(y) \cap \Gamma_{1}(z)\right| .
$$

Note that

$$
s_{i}(x, y, z)+\ell_{i}(x, y, z)=a_{i-1} .
$$

By (2.15) we have

$$
\sum_{\substack{w \in X, \partial(y, w)=i-1 \\ \partial(z, w)=1}} E \hat{w}-\sum_{\substack{w \in X, \partial(y, w)=1 \\ \partial(z, w)=i-1}} E \hat{w}=a_{i-1} \frac{\theta_{i-1}^{*}-\theta_{1}^{*}}{\theta_{0}^{*}-\theta_{i-1}^{*}}(E \hat{y}-E \hat{z}) .
$$

Taking the inner product of (3.4) with $\hat{x}$ and using (2.14) and the assumption $a_{1}=0$, we obtain

$$
s_{i}(x, y, z) \theta_{i-1}^{*}+\ell_{i}(x, y, z) \theta_{i}^{*}-a_{i-1} \theta_{2}^{*}=a_{i-1} \frac{\theta_{i-1}^{*}-\theta_{1}^{*}}{\theta_{0}^{*}-\theta_{i-1}^{*}}\left(\theta_{1}^{*}-\theta_{i}^{*}\right) .
$$

Solving $s_{i}(x, y, z)$ by using (3.3) and (3.5), we get (3.2).

By Lemma $3.1 s_{i}(x, y, z)$ is a constant for any vertices $x, y, z$ with $\partial(x, y)=1$, $\partial(y, z)=i-1, \partial(x, z)=i$.

Springer 
Definition 3.2 Let $s_{i}$ denote the expression in (3.1). Note that $s_{i}=0$ if and only if $\Gamma$ contains no parallelograms of length $i$.

Lemma 3.3 Let $\Gamma$ denote a distance-regular graph with classical parameters $(d, b, \alpha, \beta)$. Suppose that intersection numbers $a_{1}=0$ and $a_{2} \neq 0$. Then $\alpha<0$ and $b<-1$.

Proof Since $a_{1}=0$ and $a_{2} \neq 0$, from (2.19) and (2.20) we have

$$
-\alpha(b+1)^{2}=a_{2}-(b+1) a_{1}=a_{2}>0 .
$$

Hence

$$
\alpha<0 \text {. }
$$

By direct calculation from (2.17) we get

$$
\left(c_{2}-b\right)\left(b^{2}+b+1\right)=c_{3}>0 .
$$

Since

$$
b^{2}+b+1>0
$$

(3.8) implies that

$$
c_{2}>b \text {. }
$$

Using (2.17) and (3.10), we get

$$
\alpha(1+b)=c_{2}-b-1 \geq 0 .
$$

Hence, $b<-1$ by (3.7), since $b \neq-1$.

Proof of Theorem 1.1 (ii) $\Rightarrow$ (i) This is clear.

(iii) $\Rightarrow$ (ii) Suppose that $\Gamma$ has classical parameters. Then $\Gamma$ is $Q$-polynomial with associated dual eigenvalues $\theta_{0}^{*}, \theta_{1}^{*}, \ldots, \theta_{d}^{*}$ satisfying

$$
\theta_{i}^{*}-\theta_{0}^{*}=\left(\theta_{1}^{*}-\theta_{0}^{*}\right)\left[\begin{array}{l}
i \\
1
\end{array}\right] b^{1-i} \quad \text { for } 1 \leq i \leq d .
$$

We need to prove that $s_{i}=0$ for $3 \leq i \leq d$. To compute $s_{i}$ in (3.2), observe from (3.12) that

$$
\theta_{i-1}^{*}-\theta_{i}^{*}=\left(\theta_{0}^{*}-\theta_{1}^{*}\right) b^{1-i} \text { for } 1 \leq i \leq d .
$$

Summing (3.13) for consecutive $i$, we find

$$
\begin{aligned}
& \left(\theta_{1}^{*}-\theta_{i}^{*}\right)=\left(\theta_{0}^{*}-\theta_{1}^{*}\right)\left(b^{-1}+b^{-2}+\cdots+b^{1-i}\right), \\
& \left(\theta_{1}^{*}-\theta_{i-1}^{*}\right)=\left(\theta_{0}^{*}-\theta_{1}^{*}\right)\left(b^{-1}+b^{-2}+\cdots+b^{2-i}\right), \\
& \left(\theta_{2}^{*}-\theta_{i}^{*}\right)=\left(\theta_{0}^{*}-\theta_{1}^{*}\right)\left(b^{-2}+b^{-3}+\cdots+b^{1-i}\right),
\end{aligned}
$$




$$
\left(\theta_{0}^{*}-\theta_{i-1}^{*}\right)=\left(\theta_{0}^{*}-\theta_{1}^{*}\right)\left(b^{0}+b^{-1}+\cdots+b^{2-i}\right)
$$

for $3 \leq i \leq d$. Evaluating (3.2) by using (3.13-3.17), we find that $s_{i}=0$ for $3 \leq i \leq d$.

(i) $\Rightarrow$ (iii) Note that $s_{3}=0$. Then by setting $i=3$ in (3.2) and using the assumption $a_{2} \neq 0$, we find

$$
\left(\theta_{0}^{*}-\theta_{2}^{*}\right)\left(\theta_{2}^{*}-\theta_{3}^{*}\right)-\left(\theta_{1}^{*}-\theta_{2}^{*}\right)\left(\theta_{1}^{*}-\theta_{3}^{*}\right)=0 .
$$

Set

$$
b:=\frac{\theta_{1}^{*}-\theta_{0}^{*}}{\theta_{2}^{*}-\theta_{1}^{*}}
$$

Then

$$
\theta_{2}^{*}=\theta_{0}^{*}+\frac{\left(\theta_{1}^{*}-\theta_{0}^{*}\right)(b+1)}{b}
$$

Eliminating $\theta_{2}^{*}, \theta_{3}^{*}$ in (3.18) by using (3.20) and (2.16), we have

$$
\frac{-\left(\theta_{1}^{*}-\theta_{0}^{*}\right)^{2}\left(\sigma b^{2}+\sigma b+\sigma-b\right)}{\sigma b^{2}}=0
$$

for an appropriate $\sigma \in \mathbb{R} \backslash\{0\}$. Since $\theta_{1}^{*} \neq \theta_{0}^{*}$, we have

$$
\sigma b^{2}+\sigma b+\sigma-b=0
$$

and hence

$$
\sigma^{-1}=\frac{b^{2}+b+1}{b} .
$$

By Theorem 2.2, to prove that $\Gamma$ has classical parameter, it suffices to prove that

$$
\theta_{i}^{*}-\theta_{0}^{*}=\left(\theta_{1}^{*}-\theta_{0}^{*}\right)\left[\begin{array}{l}
i \\
1
\end{array}\right] b^{1-i} \quad \text { for } 1 \leq i \leq d .
$$

We prove (3.23) by induction on $i$. The case $i=1$ is trivial, and the case $i=2$ is from (3.20). Now suppose that $i \geq 3$. Then (2.16) implies

$$
\theta_{i}^{*}=\sigma^{-1}\left(\theta_{i-1}^{*}-\theta_{i-2}^{*}\right)+\theta_{i-3}^{*} \text { for } 3 \leq i \leq d .
$$

Evaluating (3.24), using (3.22) and the induction hypothesis, we find that $\theta_{i}^{*}-\theta_{0}^{*}$ is as in (3.23). Therefore, $\Gamma$ has classical parameters $(d, b, \alpha, \beta)$ for some scalars $\alpha, \beta$. Note that $b<-1$ by Lemma 3.3.

\section{Proof of Theorem 1.2}

Recall that a sequence $x, y, z$ of vertices of $\Gamma$ is geodetic whenever

$$
\partial(x, y)+\partial(y, z)=\partial(x, z) .
$$


Recall that a sequence $x, y, z$ of vertices of $\Gamma$ is weak-geodetic whenever

$$
\partial(x, y)+\partial(y, z) \leq \partial(x, z)+1 .
$$

Definition 4.1 A subset $\Omega \subseteq X$ is weak-geodetically closed if, for any weakgeodetic sequence $x, y, z$ of $\Gamma$,

$$
x, z \in \Omega \Longrightarrow y \in \Omega .
$$

Theorem 4.2 [12, Proposition 6.7, Theorem 4.6] Let $\Gamma=(X, R)$ denote a distanceregular graph with diameter $d \geq 3$. Assume that the intersection numbers $a_{1}=0$ and $a_{2} \neq 0$. Suppose that $\Gamma$ contains no parallelograms of length 3 . Then, for each pair of vertices $v, w \in X$ at distance $\partial(v, w)=2$, there exists a weak-geodetically closed subgraph $\Omega$ of diameter 2 in $\Gamma$ containing $v, w$. Furthermore, $\Omega$ is strongly regular with intersection numbers

$$
\begin{aligned}
& a_{i}(\Omega)=a_{i}(\Gamma) \\
& c_{i}(\Omega)=c_{i}(\Gamma) \\
& b_{i}(\Omega)=a_{2}(\Gamma)+c_{2}(\Gamma)-a_{i}(\Omega)-c_{i}(\Omega)
\end{aligned}
$$

for $0 \leq i \leq 2$.

Corollary 4.3 Let $\Gamma$ denote a distance-regular graph with classical parameters $(d, b, \alpha, \beta)$, where $d \geq 3$. Assume that $\Gamma$ has intersection numbers $a_{1}=0$ and $a_{2} \neq 0$. Then there exists a weak-geodetically closed subgraph $\Omega$ of diameter 2 . Furthermore, the intersection numbers of $\Omega$ satisfy

$$
\begin{aligned}
b_{0}(\Omega) & =(1+b)(1-\alpha b), \\
b_{1}(\Omega) & =b(1-\alpha-\alpha b), \\
c_{2}(\Omega) & =(1+b)(1+\alpha), \\
a_{2}(\Omega) & =-(1+b)^{2} \alpha, \\
|\Omega| & =\frac{(1+b)(b \alpha-2)(b \alpha-1-\alpha)}{(1+\alpha)} .
\end{aligned}
$$

Proof Note that $b<-1$ by Lemma 3.3 and $\Gamma$ contains no parallelograms of length 3 by Theorem 1.1. Hence there exists a weak-geodetically closed subgraph $\Omega$ of diameter 2 by Theorem 4.2. By applying (2.17), (2.18), and (2.20) to (4.1-4.3), we immediately have (4.4-4.7). Note that $|\Omega|=1+k(\Omega)+k(\Omega) b_{1}(\Omega) / c_{2}(\Omega)$. Equation (4.8) follows from this and from (4.4-4.6).

Proposition 4.4 ([12, Proposition 3.2]) Let $\Gamma$ denote a distance-regular graph with diameter $d \geq 3$. Suppose that there exists a weak-geodetically closed subgraph $\Omega$ of $\Gamma$ with diameter 2 . Then the intersection numbers of $\Gamma$ satisfy the following inequality

$$
a_{3} \geq a_{2}\left(c_{2}-1\right)+a_{1}
$$


Corollary 4.5 Let $\Gamma$ denote a distance-regular graph with classical parameters $(d, b, \alpha, \beta)$, where $d \geq 3$. Suppose that the intersection numbers $a_{1}=0$ and $a_{2} \neq 0$. Then

$$
c_{2} \leq b^{2}+b+2
$$

Proof Applying $a_{1}=0$ in (2.20), we have that $a_{3}=-\alpha\left(b^{2}+b+1\right)(b+1)^{2}$. Then by applying (4.9), using Lemma 3.3, (4.1), and (4.7), the result immediately follows.

We will decrease the upper bound of $c_{2}$ in (4.10). We need the following lemma.

Lemma 4.6 Let $\Gamma$ denote a distance-regular graph with classical parameters $(d, b, \alpha, \beta)$, where $d \geq 3$. Assume that the intersection numbers $a_{1}=0$ and $a_{2} \neq 0$. Let $\Omega$ be a weak-geodetically closed subgraph of diameter 2 in $\Gamma$. Let $r>s$ denote the nontrivial eigenvalues of the strongly regular graph $\Omega$. Then the following (i)-(ii) hold:

(i) The multiplicity of $r$ is

$$
f=\frac{(b \alpha-1)(b \alpha-1-\alpha)(b \alpha-1+\alpha)}{(\alpha-1)(\alpha+1)} .
$$

(ii) The multiplicity of $s$ is

$$
g=\frac{-b(b \alpha-1)(b \alpha-2)}{(\alpha-1)(\alpha+1)}
$$

Proof From [9, Theorem 21.1] we have

$$
\begin{aligned}
& f=\frac{1}{2}\left\{v-1+\frac{(v-1)\left(c_{2}-a_{1}\right)-2 k}{\sqrt{\left(c_{2}-a_{1}\right)^{2}+4\left(k-c_{2}\right)}}\right\}, \\
& g=\frac{1}{2}\left\{v-1-\frac{(v-1)\left(c_{2}-a_{1}\right)-2 k}{\sqrt{\left(c_{2}-a_{1}\right)^{2}+4\left(k-c_{2}\right)}}\right\},
\end{aligned}
$$

where $v=|\Omega|$, and $k$ is the valency of $\Omega$. Note that $c_{2}(\Omega)=(1+b)(1+\alpha)$ by $(2.17)$, $k(\Omega)=(1+b)(1-\alpha b)$ by $(4.4)$, and $v=(1+b)(b \alpha-2)(b \alpha-1-\alpha) /(1+\alpha)$ by (4.8). Now (4.11) and (4.12) follow from (4.13) and (4.14).

Corollary 4.7 Let $\Gamma$ denote a distance-regular graph with classical parameters $(d, b, \alpha, \beta)$, where $d \geq 3$. Assume that $\Gamma$ has intersection numbers $a_{1}=0$ and $a_{2} \neq 0$. Then

$$
\begin{gathered}
\frac{b(b+1)^{2}(b+2)}{c_{2}}, \\
\frac{(b-2)(b-1) b(b+1)}{2+2 b-c_{2}}
\end{gathered}
$$

are both integers.

를 Springer 
Proof Let $f$ and $g$ be as in (4.11-4.12). Set $\rho=\alpha(1+b)$. Note that $\rho$ is an integer, since $\rho=c_{2}-1-b$. Then both

$$
f+g-\left(1-3 b^{2}-b \rho+b^{2} \rho-b^{3}\right)=\frac{2 b+5 b^{2}+4 b^{3}+b^{4}}{1+b+\rho}=\frac{b(b+1)^{2}(b+2)}{c_{2}}
$$

and

$f-g-\left(1-3 b^{2}-b \rho+b^{2} \rho+b^{3}\right)=\frac{2 b-b^{2}-2 b^{3}+b^{4}}{-1-b+\rho}=\frac{(b-2)(b-1) b(b+1)}{c_{2}-2-2 b}$

are integers, since $f, g, b$, and $\rho$ are integers.

Proposition 4.8 Let $\Gamma$ denote a distance-regular graph with classical parameters $(d, b, \alpha, \beta)$, where $d \geq 3$. Assume that $\Gamma$ has intersection numbers $a_{1}=0$ and $a_{2} \neq 0$. Then $c_{2} \leq b(b+1)$.

Proof Recall that $c_{2} \leq b^{2}+b+2$ by (4.10). First, suppose that

$$
c_{2}=b^{2}+b+2
$$

Then the integral condition (4.15) becomes

$$
b^{2}+3 b+\frac{-4 b}{b^{2}+b+2} \text {. }
$$

Since $0<-4 b<b^{2}+b+2$ for $b \leq-5$, we have $-4 \leq b \leq-2$. For $b=-4$ or -3 , expression (4.18) is not an integer. The remaining case $b=-2$ implies $\alpha=-5$ by (4.6), $v=28$ by (4.8), and $g=6$ by (4.12). This contradicts to $v \leq \frac{1}{2} g(g+3)$ [9, Theorem 21.4]. Hence $c_{2} \neq b^{2}+b+2$. Next, suppose that $c_{2}=b^{2}+b+1$. Then (4.16) becomes

$$
-b^{2}+b+1+\frac{1}{b^{2}-b-1}
$$

It fails to be an integer, since $b<-1$.

Proof of Theorem 1.2 The results come from Corollary 4.7 and Proposition 4.8.

Example 4.9 [3] Hermitian forms graph $\operatorname{Her}_{2}(d)$ is a distance-regular graph with classical parameters $(d, b, \alpha, \beta)$ with $b=-2, \alpha=-3$, and $\beta=-\left((-2)^{d}+1\right)$, which satisfies $a_{1}=0, a_{2} \neq 0$, and $c_{2}=b(b+1)$.

Example 4.10 [9, p. 237] Gewirtz graph is a distance-regular graph with diameter 2 and intersection numbers $a_{1}=0, c_{2}=2, k=10$, which can be written as classical parameters $(d, b, \alpha, \beta)$ with $d=2, b=-3, \alpha=-2, \beta=-5$, so we have $c_{2}=\frac{(b+1)^{2}}{2}$.

Conjecture 4.11 (Gewirtz graph does not grow) There is no distance-regular graph with classical parameters $\left(d,-3,-2,-\frac{1+(-3)^{d}}{2}\right)$, where $d \geq 3$. 
There is a conjecture similar to Conjecture 4.11 for the complement part in $a_{1} \neq 0$. See [13, Theorem 10.3] for details.

Acknowledgement The authors thank Paul Terwilliger for reading the first manuscript and giving valuable suggestions.

\section{References}

1. Bannai, E., \& Ito, T. (1984). Algebraic combinatorics I: association schemes. Menlo Park: Benjamin/Cummings.

2. Brouwer, A. E., Cohen, A. M., \& Neumaier, A. (1989). Distance-regular graphs. Berlin: Springer.

3. Ivanov, A. A., \& Shpectorov, S. V. (1989). Characterization of the association schemes of Hermitian forms over $G F\left(2^{2}\right)$. Geometriae Dedicata, 30, 23-33.

4. Jurišić, A., Koolen, J., \& Miklavič, Š. On triangle-free distance-regular graphs with an eigenvalue multiplicity equal to the valency. Preprint.

5. Koolen, J. H., \& Moulton, V. (2004). There are finitely many triangle-free distance-regular graphs with degree 8, 9 or 10. Journal of Algebraic Combinatorics, 19(2), 205-217.

6. Miklavič, Š. (2004). $Q$-polynomial distance-regular graphs with $a_{1}=0$. European Journal of Combinatorics, 25(7), 911-920.

7. Terwilliger, P. (1992). The subconstituent algebra of an association scheme (Part I). Journal of Algebraic Combinatorics, 1, 363-388.

8. Terwilliger, P. (1995). A new inequality for distance-regular graphs. Discrete Mathematics, 137, 319332 .

9. van Lint, J. H., \& Wilson, R. M. (1992). A course in combinatorics. Cambridge: Cambridge University Press.

10. Weng, C. (1995). Kite-free $P$ - and $Q$-polynomial schemes. Graphs and Combinatorics, 11, $201-207$.

11. Weng, C. (1997). Parallelogram-free distance-regular graphs. Journal of Combinatorial Theory, Series $B, 71(2), 231-243$.

12. Weng, C. (1998). Weak-geodetically closed subgraphs in distance-regular graphs. Graphs and Combinatorics, 14, 275-304.

13. Weng, C. (1999). Classical distance-regular graphs of negative type. Journal of Combinatorial Theory, Series B, 76, 93-116. 